\title{
Grand Challenge in Precision Livestock Farming
}

\author{
Guilherme J. M. Rosa* \\ Department of Animal and Dairy Sciences, Department of Biostatistics \& Medical Informatics, University of \\ Wisconsin-Madison, Madison, WI, United States
}

Keywords: digital agriculture, sensor technology, computer vision, animal welfare, sustainability

\section{OPEN ACCESS}

Edited by:

Oleksiy Guzhva,

Swedish University of Agricultural

Sciences, Sweden

Reviewed by:

Albert De Vries,

University of Florida, United States

Dana L. M. Campbell,

Commonwealth Scientific and Industrial Research Organisation (CSIRO), Australia

*Correspondence:

Guilherme J. M. Rosa grosa@wisc.edu

Specialty section:

This article was submitted to Precision Livestock Farming,

a section of the journal

Frontiers in Animal Science

Received: 06 January 2021

Accepted: 21 January 2021

Published: 11 February 2021

Citation:

Rosa GJM (2021) Grand Challenge in

Precision Livestock Farming.

Front. Anim. Sci. 2:650324.

doi: 10.3389/fanim.2021.650324
Animal agriculture is a vital social and economic component of human communities, supplying them with food, labor, companionship, and raw material for a myriad of goods. The demand for animal protein and animal products is rapidly growing, given the continued world population growth as well as rising incomes and urbanization, more notably in developing countries (FAO, 2018). However, meeting such an upward demand should comply with moral and economic constraints related to animal welfare and the sustainability of agricultural enterprises while also reducing the environmental impact of animal production systems (FAO, 2017).

Precision livestock farming (PLF) proposes to address this challenge by applying technology within the animal space for automated and real-time decision making at the individual animal and group level in livestock production (Berckmans, 2017; Benjamin and Yik, 2019). Data collected by sensors (such as cameras, microphones, accelerometers, gas analyzers, and spectrometers) on animals or on their environment, coupled with advanced analytical techniques, provide efficient tools to monitor animals to improve their welfare and optimize resource use, such as feed, water, land, and human labor.

Traditionally, in livestock production, the management unit is generally a herd or flock, e.g., a group of animals in a paddock or housed together within a facility. Typical examples are large beef cattle production in either feedlots or grazing systems and modern production of pigs and poultry. Animal caretakers still try to monitor the condition of individual animals in terms of their overall health, well-being, and performance, but this is quite often a difficult task when working with large groups of animals and with limited time. Nonetheless, ideally, management practices should be efficient enough for early detection of diseases, even if subclinical, and tailored for individual animal needs to maximize animal welfare, reproductive, and production performance, as well as homogeneity within groups of pen mate animals.

Precision livestock farming technologies can alert the animal caretakers in real-time to deliver individualized care to an animal showing altered behavior as a result of disease, injury, or a stressor. PLF is also useful for many other applications to improve the efficiency of livestock operations, for e.g., to detect estrus in beef and dairy cattle for optimal reproductive management of herds, precision feeding by monitoring daily feed intake and weight gain, etc. In addition, PLF systems can measure novel phenotypes or indicator traits to be used in advanced breeding programs (Rosa, 2011). Phenotypes of economic or societal importance that are generally difficult to measure without digital technologies include, for e.g., individual feed consumption and feed efficiency, heat tolerance, mothering ability, aggressive behavior, disease resistance, among many others. Moreover, the collection of individual animal data in commercial farms provides an in-depth look at the interaction between environment (stocking density, lighting, temperature, etc.) and behavior and performance of individual animals within the space. This can help the genetic improvement of adapted animals for different environments and the development of more efficient animal facilities and equipment for enhanced animal welfare and performance.

Despite the potential of PLF to increase the efficiency and sustainability of livestock production systems, on-farm adoption of PLF is still incipient. Nonetheless, the trend is that PLF will be more 
broadly adopted as the price of sensor technologies decreases, and the animal science community develops groundbreaking basic and translational research for efficient use of PLF. The key to PLF is real-time data collection on either individual animals or groups of animals with little or no disruption of animal activities. Monitoring groups of animals or their environment requires sensors strategically positioned within their spaces (e.g., barns and paddocks), while monitoring individuals animals involves small wearable sensors integrated with animal identification and video or image analysis of the animals. Gathered data need to be processed through sophisticated machine learning and statistical analyses, which also involves efficient data management, including data transfer, storage, and editing. Lastly, independently of how modern, sophisticated, and efficient any specific PLF tool is, and its potential to improve lives of animals and animal caretakers, there is also another fundamental component for the success of PLF applications, which is the economic viability of each specific tool, and its accessibility and acceptability by farmers and farmworkers.

In this context, the development and successful application of PLF tools should involve a truly multidisciplinary approach for research and development as well as extension and outreach activities at universities, research institutions, and agriculture industries. To deal with such complexities and challenges posed by PLF, a joint effort is required by individuals from various disciplines and expertise, including animal scientists and veterinarians, electrical engineers and computer scientists, data scientists, agricultural and biological systems engineers, economists, and sociologists, among others.

Certainly, there are still many challenges that must be tackled so that PLF can be more widely used for different livestock species, and different production systems. First, PLF tools should be designed according to the reality of each country or global region, not only in terms of the specific climate and weather conditions, but also farm sizes and technological level, labor availability and labor training, market requirements, etc. For example, while electricity, Internet, and cell phone signal may be more broadly available in rural areas of developed countries, this is certainly not the case in many developing regions of the world. Also, countries can have vastly different labor force participation rates in agriculture, so that PLF and farm automation can affect them differently.

Another challenge for PLF is the continuous development of efficient and reliable sensors (in terms of sensitivity to gather the intended signal, durability, cost, miniaturization, and efficiency of power utilization) as well as Internet of Things (IoT) systems to integrate PLF tools and to provide efficient data transmission and data storage, including data security and privacy. All this requires dedicated professionals in the areas of electrical engineering and computer science, biological systems engineering, among others, with a good understanding of farm production systems and livestock biology.

The potential amount of data generated through the use of sensors and camera technologies is unprecedented. Sensor technologies can generate multiple data points per animal daily. For example, consider a PLF device based on a camera sensor capturing a single image of 5 megabytes every second from a pen or individual animal. In a single day, there would be 86,400 images for a total of 432 gigabytes, or about 13 terabytes per month. This definitely poses a tremendous challenge from a data transmission and data storage standpoint, and so computer science expertise is essential to optimize such systems.

In terms of data analysis, careful data editing and quality control protocols, and sophisticated machine learning algorithms and statistical models are required to deal with such multidimensional datasets, involving complex interdependencies between interacting variables measured over time, including missing data and potential recording errors, confounding effects, and redundancies. Most applications of sensor technologies involve a prediction task, so techniques of machine learning and pattern recognition, time series, and cross-validation are extremely important. The power of information in more complex data streams, as in computer vision systems, has been harvested through deep learning algorithms, which have been reported as extremely useful analytical tools (Fernandes et al., 2020).

Lastly, another critical component of PLF is how to best utilize the additional information and insight provided by sensors and related predictive algorithms for optimal data-driven decisions. Some PLF applications are straightforward in this regard, for e.g., sensors used for animal identification or heat detection in cattle for insemination decisions. Other applications, however, will provide an opportunity for a complete paradigm shift in terms of animal care. For example, years of research in veterinary medicine allowed the development of efficient protocols for treatment of animals diagnosed with specific diseases, including for example the choice of antibiotics, the appropriate dose and treatment period. Suppose now that a PLF tool allows the detection of a particular disease a few days in advance, potentially at its very early stages of development. This may provide a huge opportunity to develop new protocols of preventive medicine and treatment, to minimize the impact on the individual and to prevent or slow the spread of the disease through the herd/flock. More importantly, the earlier detection of diseases may reduce the antibiotic doses or even motivate the development of antibiotic-free treatments.

Another example is in the context of feeding. Nowadays, most commercial livestock operations manage animals on a group (or pen) basis only, with diet composition and feed delivery tailored for the group average. However, due to animal-to-animal variation, some individuals may be underfed while others may be overfed. Nonetheless, with the advent of PLF technology that allows monitoring of individual animal feed intake as well as body weight gain and development, diets might be mixed in real-time and delivered precisely according to each animal's need, maximizing animal performance, and minimizing feed losses (Pomar and Remus, 2019). PLF advancements will then contribute decisively to the implementation of optimal "individualized" treatment of animals, improving upon the traditional "pen-alyzed" approach based on the group average.

Surely, PLF tools will open new avenues for a myriad of opportunities for advances in livestock production and animal 
care, which will only be fully accomplished with dedicated and innovative research. This is precisely the main goal of Frontiers in Animal Science | Precision Livestock Farming (PFL), i.e., to provide an open forum for dissemination and discussion of scientific research findings on the development and application of PFL tools to advance the efficiency of livestock systems while improving animal welfare and reducing the environmental footprint of animal agriculture. Manuscripts contributing to the advance of any aspect of PLF are welcome, including hypothesis-driven research in precision feeding, reproduction, and overall management of animals and animal systems, animal behavior and well-being, as well as research on social and economic implications of PLF adoption, the development of

\section{REFERENCES}

Benjamin, M., and Yik, S. (2019). Precision livestock farming in swine welfare: a review for swine practitioners. Animals 9:133. doi: 10.3390/ani9040133

Berckmans, D. (2017). General introduction to precision livestock farming. Anim. Front. 7:6. doi: 10.2527/af.2017.0102

FAO (2017). Livestock Solutions for Climate Change. FAO. Available online at: http://www.fao.org/3/a-i8098e.pdf

FAO (2018). "Shaping the future of livestock sustainably, responsibly, efficiently," in The 10th Global Forum for Food and Agriculture. Berlin: FAO. Available online at: http://www.fao.org/3/i8384en/I8384EN.pdf

Fernandes, A. F. A., Dórea, J. R. R., and Rosa, G. J. M. (2020). Image analysis and computer vision applications in animal sciences: an overview. Front. Vet. Sci. 7:551269. doi: $10.3389 /$ fvets.2020.551269

Pomar, C., and Remus, A. (2019). Precision pig feeding: a breakthrough toward sustainability. Anim. Front. 9, 52-55. doi: 10.1093/af/vfz006 innovative technologies and novel statistical and computational algorithms for implementing PLF tools.

\section{AUTHOR CONTRIBUTIONS}

The author confirms being the sole contributor of this work and has approved it for publication.

\section{ACKNOWLEDGMENTS}

The author acknowledges support from the Wisconsin Agriculture Experiment Station Hatch grant (142-AAC7939) and the United States Department of Agriculture (USDA).

Rosa, G. J. M. (2011). Grand challenge in livestock
genomics: for food, for medicine, for the environment,
for knowledge. Front. Gene. 2:34. doi: $10.3389 /$ fgene.2011.0
0034

Conflict of Interest: The author declares that the research was conducted in the absence of any commercial or financial relationships that could be construed as a potential conflict of interest.

Copyright (c) 2021 Rosa. This is an open-access article distributed under the terms of the Creative Commons Attribution License (CC BY). The use, distribution or reproduction in other forums is permitted, provided the original author(s) and the copyright owner(s) are credited and that the original publication in this journal is cited, in accordance with accepted academic practice. No use, distribution or reproduction is permitted which does not comply with these terms. 